\title{
EFEITO DO NÚMERO DE RAMOS PRODUTIVOS SOBRE O DESENVOLVIMENTO DA ÁREA FOLIAR E PRODUTIVIDADE DA FIGUEIRA ${ }^{1}$
}

\author{
LUIZ CARLOS SANTOS CAETANO ${ }^{2}$, ALMY JUNIOR CORDEIRO DE CARVALHO ${ }^{3}$, ELIEMAR CAMPOSTRINE ${ }^{3}$, \\ ELIAS FERNANDES DE SOUSA ${ }^{3}$, KATIA REGINA NAOMI MURAKAMI ${ }^{4}$, BRUNO SALES CEREJA ${ }^{4}$
}

\begin{abstract}
RESUMO - O objetivo deste trabalho foi estudar o efeito do número de ramos produtivos sobre o desenvolvimento da área foliar e produtividade de figos verdes do cultivar Roxo de Valinhos. O experimento utilizando plantas de seis anos de idade, foi conduzido de novembro de 2002 a abril de

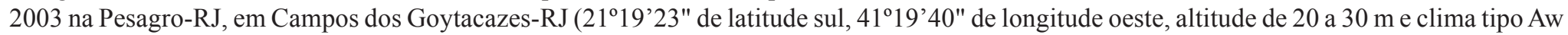
Köppen). Os tratamentos consistiram de plantas conduzidas com 16; 20; 24; 28 e 32 ramos. Utilizou-se do delineamento experimental de blocos ao acaso e cinco repetições. O IAF aumentou linearmente em função do número de ramos produtivos, enquanto a produtividade mostrou um modelo de resposta quadrática aos tratamentos, provavelmente, limitada pelo sombreamento no interior do dossel que reduz a formação de gemas frutíferas. A maior produtividade observada de figos verdes foi obtida quando as plantas foram conduzidas com 24 ramos, sendo que, neste tratamento, a área foliar média de cada planta foi de $6,2 \mathrm{~m}^{2}$.
\end{abstract}

Termos para indexação: Ficus carica, figo verde, estrutura da copa, poda.

\section{EFFECT OF THE NUMBER OF PRODUCTIVE BRANCHES ON THE LEAF AREA DEVELOPMENT AND FIG TREE YIELD}

\begin{abstract}
The objective of the present work was to study the effect of number of productive branches on the leaf area development and green fig tree yield of Roxo de Valinhos cultivar. The experiment using six year-old plants was carried out between November/2002 and April/2003 at Pesagro-RJ, Campos dos Goytacazes, RJ ( $21^{\circ} 19^{\prime} 23^{\prime \prime}$ South latitude, $41^{\circ} 19^{\prime} 40^{\prime \prime}$, West longitude, altitude of 20 a $30 \mathrm{~m}$ and Aw Koppen climate). The treatments consisted of plants conducted with 16, 20, 24, 28 and 32 productive branches in a randomized block design with five replicates. The LAI incresead linearly in function of productive branches, whereas the yield showed a quadratic response pattern to the treatments, probably, limited by shading in canopy that decrease the fruit bud formation. The highest green fig yield was observed in the treatment which had plants conducted with twenty four branches, where the mean leaf area was $6,2 \mathrm{~m}^{2}$.
\end{abstract}

Index terms: Ficus carica, green fig, canopy structure, pruning.

\section{INTRODUÇÃO}

Nos projetos com a cultura da figueira implantados nas diversas regiões do Estado do Rio de Janeiro, tem surgido o questionamento por parte de produtores e técnicos de qual seria o número de ramos adequados para a condução das figueiras, uma vez que o número de ramos produtivos tem uma relação estreita com a produtividade.

Pereira (1981) recomenda que, para a produção de figos de mesa (maduros), as plantas sejam formadas com 12 ramos e que, para a produção exclusiva de figos verdes para indústria, as plantas podem ser conduzidas com 20 a 30 ramos. Regitano (1957) indica que, para a produção exclusiva de figos verdes, as plantas podem ser conduzidas com 25 a 35 ramos. O autor constatou, em trabalho realizado na região de Campinas-SP, que à medida que se aumentava o número de ramos por planta, ocorria aumento na produção e no número de frutos. Verificou também que, quanto maior o número de ramos, menor era o peso do fruto maduro, o que não ocorria com os frutos verdes.

Dos fatores envolvidos na produtividade agrícola, a fotossíntese é o mais determinante. A elevação das taxas de fotossíntese depende, dentre outros fatores, do máximo aproveitamento da luz disponível, o qual pode ser obtido pela manipulação cultural. As formas de manipulação cultural compreendem uma população de plantas adequada ao objetivo da exploração, arranjos foliares mais erectófilos, disposição das linhas de plantio na direção norte-sul e técnicas de manejo da copa, tais como podas, desfolhamento e modificação da arquitetura da planta (Jackson, 1980; Bernardes, 1987).

Em fruteiras, a determinação da melhor arquitetura do dossel de uma planta está intimamente relacionada à otimização da distribuição da luz para formação de gemas frutíferas e qualidade de frutos ao invés de uma maximização da interceptação e produção de matéria seca - maximização do índice de área foliar (IAF) (Jackson, 1980; Lucchesi, 1987).

O objetivo deste trabalho foi determinar o número mais adequado de ramos produtivos para a condução da figueira por meio de avaliações da produção de frutos verdes e avaliar o desenvolvimento da área foliar e sua influência sobre os componentes da produção.

\section{MATERIAL E MÉTODOS}

O experimento foi conduzido na Estação Experimental da Pesagro-RJ, em Campos dos Goytacazes-RJ (21¹9'23" de latitude sul e $41^{\circ} 19^{\prime} 40^{\prime \prime}$ de longitude oeste), com plantas da cultivar Roxo de Valinhos de seis anos de idade, cultivadas em um cambissolo e no espaçamento 3 x 1,5 m. A altitude do município varia de 20 a $30 \mathrm{~m}$, e o clima é do tipo Aw de Köppen. A poda das plantas foi realizada em outubro de 2002. Cerca de trinta dias depois, selecionou-se o número de ramos desejados para a composição dos tratamentos $(16 ; 20 ; 24 ; 28$ e 32 ramos $)$. As colheitas foram realizadas entre janeiro e abril de 2003.

$\mathrm{O}$ experimento foi instalado em delineamento estatístico de blocos casualizados, com cinco repetições. Quando foram consideradas as épocas de avaliação na análise estatística, os tratamentos de número de ramos compuseram as parcelas e as épocas de avaliação, as subparcelas, compondo esquema de parcelas subdivididas no tempo. As parcelas experimentais foram compostas por três plantas em linha, sendo útil a planta central. Os dados obtidos foram submetidos à análise de variância e de regressão. Nos testes F e de Tukey, o nível de probabilidade utilizado foi de $5 \%$.

Em cada planta útil, dois ramos foram marcados, tomando-se individualmente sua produção, o número de frutos produzidos, o comprimento dos ramos, o número de nós e a área foliar. Os dados de produção e de número de frutos foram apresentados de forma totalizada.

\footnotetext{
(Trabalho 043/2005). Recebido: 11/03/2005. Aceito para publicação: 08/12/2005.

${ }^{2}$ Eng. Agrônomo, DSc., Pesquisador do Incaper, Rua Dom Fernando 39, Independência, Cachoeiro de Itapemirim-ES, 29.306-350, luizcaetano@incaper.es.gov.br.

${ }^{3}$ Eng. Agrônomo, DSc, Professor, Universidade Estadual do Norte Fluminense, Av. Alberto Lamego, 2000, Horto, Campos dos Goytacazes-RJ, 28.015-620, almy@uenf.br, campost@uenf.br, efs@uenf.br.

${ }^{4}$ Eng. Agrônomo, Bolsista, Universidade Estadual do Norte Fluminense, Av. Alberto Lamego, 2000, Horto, Campos dos Goytacazes-RJ, 28.015-620, cereja@uenf.br.
} 
O comprimento de ramos, o número de nós e a área foliar foram avaliados em quatro épocas: 14 de janeiro, 11 de fevereiro, 12 de março e 09 de abril de 2003, respectivamente, aos 90; 120; 150 e 180 dias após a poda. Da produção dos ramos marcados, determinou-se o peso médio de frutos. O comprimento dos ramos era tomado com um barbante de algodão, o qual era medido posteriormente com uma trena.

Foram também determinados o número total de frutos e a produtividade. O número total de frutos por tratamento foi obtido pela soma do número de frutos do respectivo tratamento obtidos em cada colheita. O número de frutos por tratamento em cada colheita foi determinado pela divisão do peso total de frutos daquele tratamento pelo correspondente peso médio dos frutos dos ramos marcados. Os frutos foram colhidos no ponto adequado para industrialização do fruto verde, ou seja, no máximo crescimento, com coloração da casca verde intenso e partes internas com coloração branca.

Para a determinação da área de cada folha, utilizou-se do modelo matemático $\mathrm{AF}=0,8414 \mathrm{C}^{2}$, onde $\mathrm{AF}$ é a área foliar $\left(\mathrm{cm}^{2}\right)$ e C o comprimento de cada folha (cm) medido ao longo da nervura central (Caetano, 2004). A área foliar de cada planta útil foi obtida pelo produto da área foliar média dos ramos marcados pelo número de ramos do tratamento correspondente. O IAF (índice de área foliar) foi calculado pela divisão da área foliar total da planta pela área ocupada pela mesma no espaçamento adotado $\left(4,5 \mathrm{~m}^{2}\right)$.

A avaliação da penetração de luz dentro da copa foi realizada com um medidor portátil de fluxo de fótons fotossintéticos (FFF), modelo LI 189, Li-Cor, Nebraska, USA. Os valores de penetração de luz (FFF) no dossel das plantas foram obtidos da média de vinte leituras tomadas sob o dossel de folhas em cada planta útil acerca de $30 \mathrm{~cm}$ do solo, no dia 04 de fevereiro de 2004, entre 14 e $15 \mathrm{~h} 30$.

\section{RESULTADOS E DISCUSSÃO}

A análise de regressão mostrou comportamento quadrático da produtividade em função do número de ramos produtivos conduzidos, com máxima estimada em 10,8 t/ha, com 27 ramos conduzidos (Figura 1). Porém, a maior produtividade observada, 11,9 t/ha, foi verificada no tratamento em que as plantas foram conduzidas com 24 ramos.

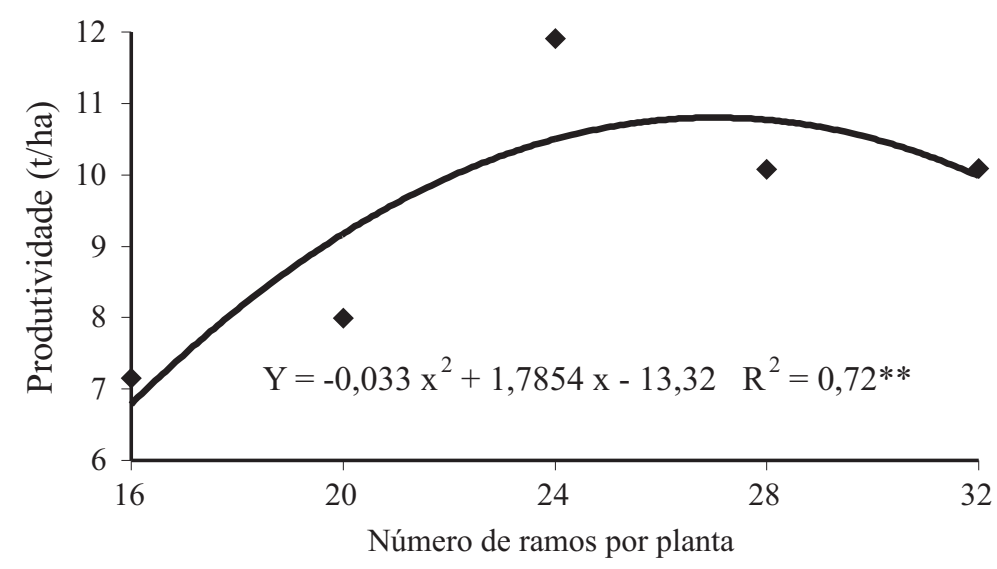

FIGURA 1 - Produtividade da figueira em função do número de ramos produtivos conduzidos.

Os resultados de produtividade podem ser explicados pelo número total de frutos produzidos (Figura 2), já que o peso médio de frutos, com média geral de $12 \mathrm{~g}$, não foi influenciado pelos tratamentos $(\mathrm{F}>0,05)$. No tratamento com 24 ramos, obteve-se a produção de maior número de frutos: 426 por planta. A análise de regressão apresentou comportamento quadrático com máximo de 407 frutos, com 29 ramos conduzidos.

O número médio de frutos/ramo apresentou comportamento quadrático (Figura 3). Estes resultados indicam que, provavelmente, a elevação do sombreamento na parte produtiva das plantas, com o aumento do número de ramos conduzidos, induziu diminuição no número

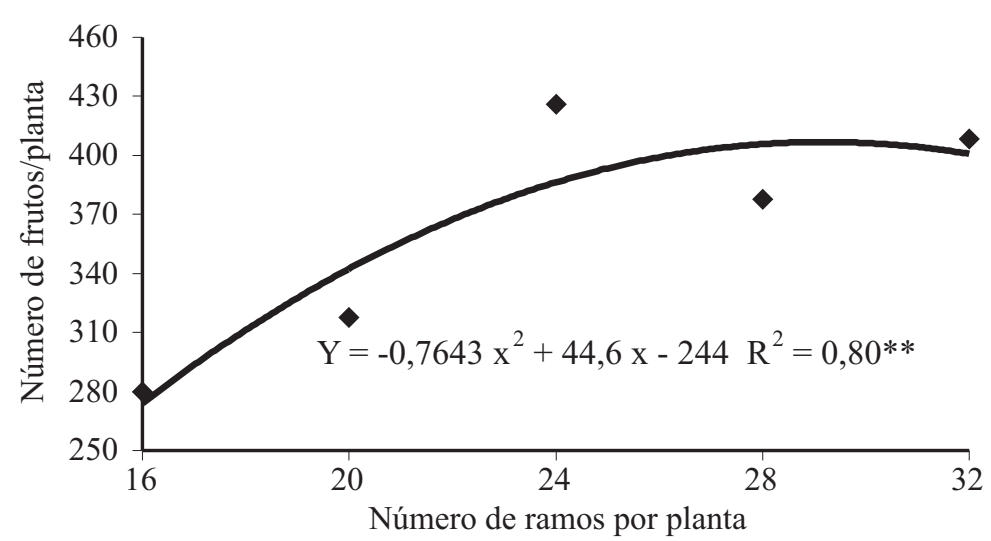

FIGURA 2 - $\mathrm{N}^{\circ}$ total de frutos produzidos por planta em função do número de ramos produtivos conduzidos.

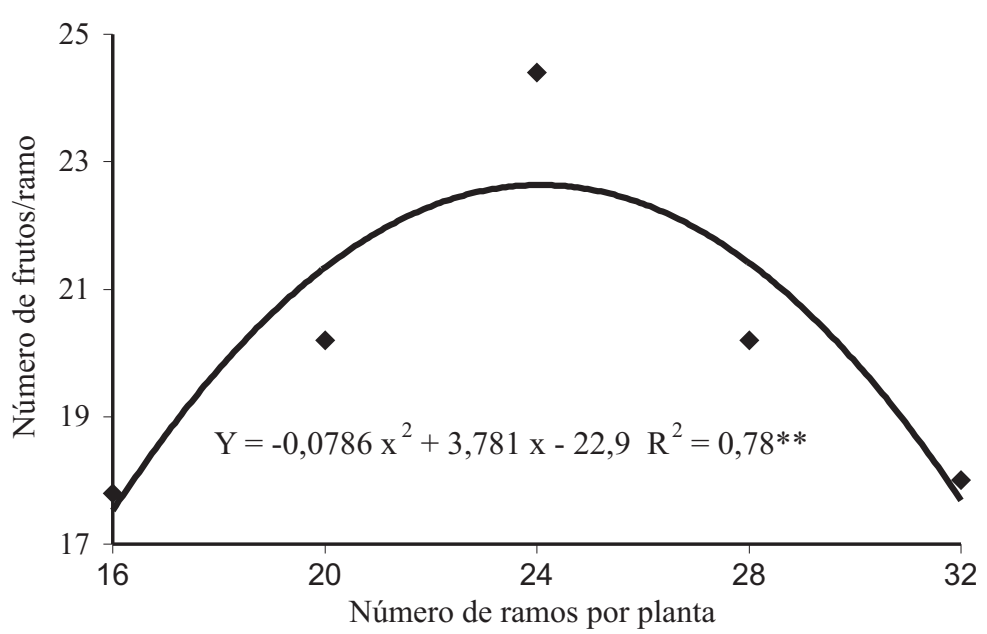

FIGURA 3 - № de frutos por ramo em função do número de ramos produtivos conduzidos.

de frutos produzidos, o que deve ser resultado da redução do número de gemas reprodutivas (não diferenciação das gemas), uma vez que o número de nós dos ramos não foi influenciado pelo número de ramos conduzidos por planta $(\mathrm{F}>0,05)$. As figueiras apresentam as gemas frutíferas localizadas nos nós dos ramos (Pereira \& Nachtigal, 1999). Em macieiras, resultados de pesquisas mostraram que o aumento da intensidade de luz no interior do dossel estimula a formação de gemas frutíferas, enquanto o sombreamento apresenta efeitos opostos (Jackson, 1980).

A penetração da radiação na copa das figueiras foi avaliada nas leituras do fluxo de fótons fotossintéticos $\left(\mu \mathrm{mol} . \mathrm{m}^{2} . \mathrm{s}^{-1}\right)$. Observouse redução linear no fluxo de fótons fotossintéticos $(\mathrm{Y}=-60,192 \mathrm{X}+$ $\left.2321, \mathrm{R}^{2}=0,94 * *\right)$ no interior da copa das plantas com o aumento do número de ramos produtivos conduzidos, o que indica a ocorrência de auto-sombreamento.

A luz é importante para a produção de frutos, pois todos os aspectos do crescimento da planta e dos frutos e o desenvolvimento de gemas floríferas requerem carboidratos que são produzidos pela fotossíntese nas folhas (Rajapakse et al.,1999; Marini, 2002). Na transição para o florescimento, ocorre aumento no suprimento de carboidratos nas gemas vegetativas, pelo aumento da atividade fotossintética e hidrólise do amido. A sacarose é acumulada no meristema para o fornecimento de energia para o processo de ativação mitótica (Bodson \& Outlaw, 1985).

O sombreamento leva ainda à redução da fotossíntese total da planta, pois folhas sombreadas apresentam menor taxa fotossintética e assim contribuem menos ou negativamente para a produção da planta que folhas expostas ao sol. Folhas de sol caracterizam-se, entre outras, pelo maior teor de clorofila e rubisco, maior densidade estomática, menor área foliar e maior peso de folha por superfície (Bernardes, 1987; Larcher, 2000). Desta maneira, nos tratamentos com maior grau de autosombreamento, espera-se redução na produtividade. 
$\mathrm{Na}$ cultura da uva, foi observado que a luz solar incidente sobre as gemas, a luminosidade diária e a temperatura foram os principais fatores climáticos que atuaram sobre o aumento da diferenciação floral (Leão \& Silva, 2003). Sandri et al. (2003) estudaram em cultivo protegido sob túnel alto o efeito do sombreamento (tela com $52 \%$ de sombreamento) sobre a produção do tomateiro, encontrando redução no número de frutos produzidos nestas condições em relação ao controle ( $83 \%$ de transmissividade)

O comprimento dos ramos da figueira não foi influenciado pelo número de ramos conduzidos. Observou-se, também, incremento no comprimento dos ramos e do número de nós/ramo da primeira para a quarta época de avaliação (Figura 4).

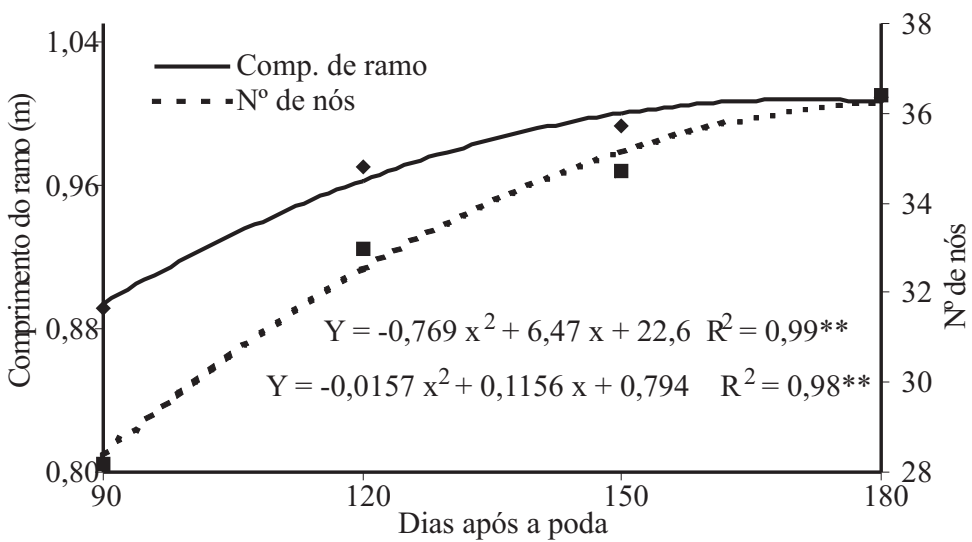

FIGURA 4 - Comprimento médio dos ramos em função da época de avaliação, em dias após a poda.

Não ocorreu diferença significativa para a área foliar média dos ramos entre os tratamentos, nas quatro avaliações realizadas (Tabela 1). Os resultados indicam que, neste experimento, o auto-sombreamento não influenciou significativamente na área das folhas, já que as folhas de sombra normalmente são maiores e mais finas (Bernardes, 1987). Em decorrência destes resultados, considerou-se a área foliar total de cada planta como o produto do número de ramos produtivos de cada planta (tratamento) pela área foliar média dos ramos. Os dados assim gerados e submetidos à análise de variância e regressão mostraram que a área foliar total da planta $\left(\mathrm{Y}=0,2624 \mathrm{X}+0,0564, \mathrm{R}^{2}=0,99 * *\right)$ e o IAF $(\mathrm{Y}=$ $\left.0,0583 \mathrm{X}+0,0125, \mathrm{R}^{2}=0,99^{* *}\right)$ aumentaram linearmente com o número de ramos produtivos conduzidos. Como já foi apresentado, os componentes da produção não apresentaram o mesmo comportamento encontrado para a área foliar. A produção fotossintética não aumenta indefinidamente com o IAF, sendo limitada pelo sombreamento que as folhas superiores exercem sobre as inferiores. $\mathrm{O}$ auto-sombreamento no dossel provoca decréscimo na taxa fotossintética média em função do aumento do IAF e reduz a formação de gemas reprodutivas (Jackson, 1980; Bernardes, 1987; Lucchesi, 1987).

Uma vez que a interação época de avaliação x número de ramos não foi significativa (teste de $\mathrm{F}$ ao nível de $5 \%$ de probabilidade), conclui-

TABELA 1 - Área foliar $\left(\mathrm{m}^{2}\right)$ dos ramos em função do número de ramos produtivos conduzidos e da época de avaliação (dias após a poda)

\begin{tabular}{ccccc}
\hline Número de ramos produtivos & \multicolumn{5}{c}{ Área foliar/ramo (1) } \\
\cline { 2 - 5 } & 90 & 120 & 150 & 180 \\
\hline & 0,31 & 0,29 & 0,25 & 0,24 \\
20 & 0,34 & 0,28 & 0,21 & 0,21 \\
24 & 0,32 & 0,25 & 0,24 & 0,21 \\
28 & 0,35 & 0,27 & 0,21 & 0,22 \\
32 & 0,32 & 0,29 & 0,23 & 0,23 \\
\hline Média & 0,33 & 0,28 & 0,23 & 0,22 \\
\hline CV (\%) & \multicolumn{5}{c}{22,44} \\
\hline
\end{tabular}

(1) Regressões não significativas. se que a área foliar média de $6,20 \mathrm{~m}^{2}$ foi a mais adequada para figueira nas condições deste experimento, já que corresponde ao tratamento no qual se observou a maior produtividade ( 24 ramos produtivos).

Considerando todos os tratamentos, a área foliar média dos $\operatorname{ramos}\left(\mathrm{Y}=-0,0367 \mathrm{X}+0,3548, \mathrm{R}^{2}=0,93 * *\right)$ e a área foliar total $(\mathrm{Y}=-0,9052$ $\left.\mathrm{X}+8,618, \mathrm{R}^{2}=0,92 * *\right)$ das plantas diminuíram a partir da primeira época de avaliação, sendo os valores da área foliar média dos ramos e da área foliar total da planta de, respectivamente: $0,33 \mathrm{~m}^{2}$ e $7,89 \mathrm{~m}^{2}$ na primeira avaliação, 0,28 $\mathrm{m}^{2}$ e $6,76 \mathrm{~m}^{2}$ na segunda avaliação, $0,23 \mathrm{~m}^{2}$ e 5,45 $\mathrm{m}^{2}$ na terceira avaliação e $0,22 \mathrm{~m}^{2}$ e $5,31 \mathrm{~m}^{2}$ na quarta avaliação. A abscisão natural de folhas, quando as plantas vão chegando ao final do ciclo produtivo, e, principalmente, o aumento da incidência da ferrugem da figueira (Cerotelium fici) é que causaram a redução da área foliar das plantas ao longo das avaliações.

A poda insuficiente em fruteiras resulta em aumento da estrutura vegetativa da planta. Este crescimento causa pesado sombreamento, e as gemas frutíferas param de se desenvolver. Desta forma, em poucos anos, as gemas frutíferas se desenvolverão somente no topo e lados da planta onde há incidência da luz solar. Podando-se uma larga porção das folhas e ramos dentro da copa, aumenta a penetração da luz solar, que promoverá o desenvolvimento de gemas frutíferas no interior da área da planta (Kadir, 2003). A utilização da poda permite, portanto, adequar a copa da planta para obtenção de máxima produtividade e qualidade do produto colhido.

\section{CONCLUSÕES}

$\mathrm{O}$ aumento do número de ramos conduzidos não elevou a produtividade de figos verdes de forma crescente, pois o autosombreamento proporcionado por uma estrutura de copa com mais ramos diminuiu o número frutos formados. A maior produtividade observada de figos verdes foi obtida quando as plantas foram conduzidas com 24 ramos, sendo que, neste tratamento, a área foliar média de cada planta foi de $6,2 \mathrm{~m}^{2}$. Não ocorreu diferença significativa para a área foliar média dos ramos entre os tratamentos em quatro avaliações realizadas, mas a área foliar total da planta e o IAF aumentaram linearmente com o número de ramos produtivos conduzidos, porém ocasionando sombreamento dentro da copa nos tratamentos com maior número de ramos.

\section{REFERÊNCIAS}

BERNARDES, M.S. Fotossíntese no dossel das plantas cultivadas. In: CASTRO, P.R.C., FERREIRA, S.O., YAMADA, T. (Eds). Ecofisiologia da produção agrícola. Piracicaba: Associação brasileira para pesquisa da potassa e do fosfato, 1987. p.13-45.

BODSON, M.; OUTLAW Jr., W. Elevation in sucrose content of the shoot apical meristema of sinapis albaat floral evocation. Plant Physiology, Maryland, v.79, n.2, p.20-24, 1985.

CAETANO, L.C.S. Sistemas de condução, nutrição mineral e adubação da figueira "Roxo de Valinhos" na região Norte Fluminense. 2004. 106 f. Dissertação (Doutorado em Produção Vegetal) - Universidade Estadual do Norte Fluminense, Rio de Janeiro. 2004.

JACKSON, J.E. Light interception and utilization by orchard systems. Horticultural Reviews, New York, v.2, p.208-267, 1980.

KADIR, S. Why fruit trees fail to bear. Kansas: Kansas State University, 2003. 4p. (Horticultural Report).

LARCHER, W. Ecofisiologia vegetal. São Carlos: RiMa, 2000. 531p.

LEÃO, P.C.S.; SILVA, E.E.G. Brotação e fertilidade de gemas em uvas sem sementes no Vale do São Francisco. Revista Brasileira de Fruticultura, Jaboticabal, v.25, n.3, p.1-7, 2003.

LUCCHESI, A.A. Fatores da produção vegetal. In: CASTRO, P.R.C., FERREIRA, S.O., YAMADA, T. (Eds). Ecofisiologia da produção agrícola. Piracicaba: Associação brasileira para pesquisa da potassa e do fosfato, 1987, p.1-11.

MARINI, R.Tree management for improving peach fruit quality. In:Mid 
atlantic fruit vegetable convention, 2002. Disponível em: $<$ http:// www.rce.rutgers.edu/peach/orchard/ treemanegement $>$. Acesso em: 15 out. 2003.

PEREIRA, F.M.; NACHTIGAL, J.C. Botânica, biologia e cultivares de figueira. In: CORREAA, L. DE S.; BOLIANI, A.C. Cultura da figueira: do plantio à comercialização. Ilha Solteira: FUNEP, 1999. p.25-35.

PEREIRA, F.M. Cultura da figueira. Piracicaba: LivroCeres, 1981.73p.

RAJAPAKSE, N.C.; YOUNG, R.E.; MCMAHON, M.J.; Oi, R. Plant height control by photoselective filters: current status and future prospects. HortTechnology, Alexandria, v.9, n.4, p.618-624, 1999.
REGITANO, O. Resultados experimentais relativos à poda da figueira, variedade "Roxo de Valinhos". Bragantia, Campinas, v.16, n.9, p.109-130, 1957.

SANDRI, M.A.; ANDRIOLO, J.L.; WITTER, M.; DAL ROSS, T. Effect of shading on tomato plant grow under greenhouse. Horticultura Brasileira, Brasília, v.21, n.4, p.642-645, 2003. 